Classification

Physics Abstracts

$41.10 \mathrm{~F}$

\title{
Minimisation des inductances propres des condensateurs à film métallisé
}

\author{
Ch. Joubert, G. Rojat et A. Béroual \\ CEGELY (URA 829 CNRS), Ecole Centrale de Lyon, BP 163, 69131 Ecully Cedex, France
}

(Reçu le 20 octobre 1994, révisé le 20 mars 1995, accepté le 24 mars 1995)

Résumé. -- Dans cet article nous examinons les différents facteurs responsables de l'inductance équivalente série dans les condensateurs à film métallisé et proposons des géométries de condensateurs qui réduisent cette inductance. Après avoir rappelé la structure des condensateurs à film métallisé, nous comparons, par des mesures expérimentales, l'inductance due au bobinage et l'inductance ajoutée par les connexions. Cette dernière peut devenir prépondérante. Afin d'expliquer l'évolution de l'impédance du bobinage en fonction de la fréquence, nous décrivons un modèle analytique qui donne la densité du courant dans le bobinage et l'impédance de ce dernier. En outre, ce modèle permet de déterminer la fréquence de résonance série de divers types de condensateurs ce qui permet de déduire l'influence de la hauteur des condensateurs et de leurs rayons interne et externe sur les performances. Il apparaît ainsi que, pour diminuer l'inductance équivalente série, il vaut mieux employer des bobinages plats et des bobinages annulaires.

\begin{abstract}
In this article, we examine the different factors responsible for the equivalent series inductance in metallized capacitors and we propose structures for capacitors that reduce this inductance. After recalling the structure of metallized capacitors we compare, by experimental measurements, the inductance due to the winding and that one added by the connections. The latter can become preponderant. In order to explain the experimental evolution of the winding impedance vs. frequency, we describe an analytical model which gives the current density in the winding and its impedance. This model enables us to determine the self resonant frequency for different types of capacitors. From where, we can infer the influence of the height of capacitors and their internal and external radius upon performances. It appears that to reduce the equivalent series inductance, it is better to use flat windings and annular windings.
\end{abstract}

\section{Introduction}

Pour limiter le poids et le volume des composants passifs magnétiques - inductances et transformateurs - et des condensateurs, les concepteurs de systèmes d'électronique de puissance augmentent la fréquence de travail des interrupteurs. Cette évolution fait apparaître des contraintes supplémentaires sur l'ensemble des composants du convertisseur, en particulier sur les condensateurs employés comme dispositifs de filtrage et de découplage, et comme circuits d'aide à la 
commutation (CALC). Ces condensateurs doivent en effet garder une impédance faible malgré l'augmentation de la fréquence, et induire le moins d'oscillations parasites possible pour réduire les perturbations électromagnétiques. En conséquence, leur inductance série équivalente doit être minimisée. Cette inductance est due, d'une part, aux connexions entre le bobinage $\mathrm{du}$ condensateur et le dispositif d'électronique de puissance et, d'autre part, à l'inductance propre du bobinage. L'objet de cet article est de présenter les résultats d'une étude concernant l'influence des éléments constitutifs du condensateur sur l'inductance totale. Seront successivement étudiées dans cet article, la structure du condensateur et l'influence des connexions mise en évidence par des mesures d'impédance du condensateur complet et du bobinage seul. La répartition du courant dans le bobinage et l'influence de la géométrie du bobinage seront également traitées à l'aide de simulations numériques. Nous discuterons enfin des géométries préférentielles pour la conception du bobinage.

\section{Structure du condensateur}

Les condensateurs à film métallisé sont constitués de deux films diélectriques enroulés sur un mandrin isolant de rayon $R_{\text {int }}[1,2]$. Le rayon extérieur du bobinage terminé est noté $R_{\text {ext }}$. Une seule face de chaque film est métallisée et constitue une électrode du condensateur. Pour pouvoir établir facilement une connexion électrique avec le circuit extérieur, les deux films sont légèrement décalés l'un par rapport à l'autre. On projette ensuite du métal fondu à chaque extrémité de l'enroulement (procédé de "shoopage"). On peut alors souder un fil électrique de liaison avec les bornes sur chaque extrémité. Un de ces fils passe soit à l'intérieur du mandrin soit à l'extérieur pour assurer le retour du courant (Fig. 1). Nous supposerons par la suite que l'axe $\left(O, \mathbf{e}_{z}\right)$ est un axe de symétrie de révolution du condensateur. En conséquence, les grandeurs physiques ne dépendent que de $r$ et de $z$. De plus, on peut noter que cette symétrie permet d'annuler le champ magnétique à l'extérieur du bobinage et donc de minimiser les perturbations électromagnétiques dues au condensateur.

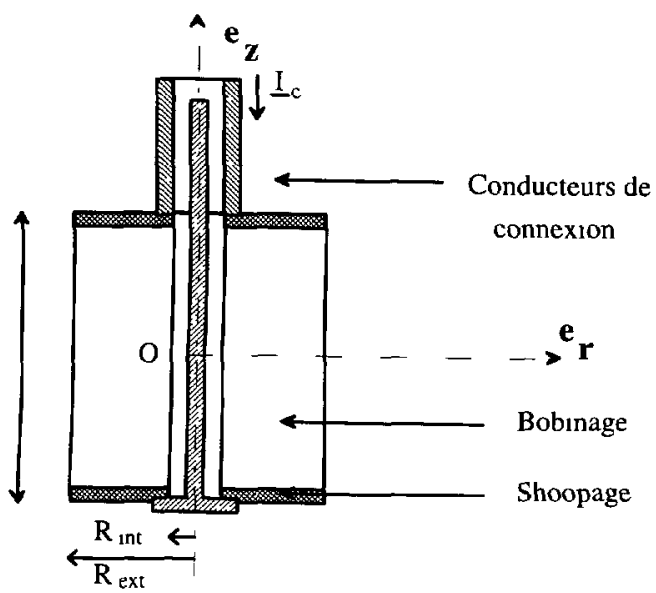

a)

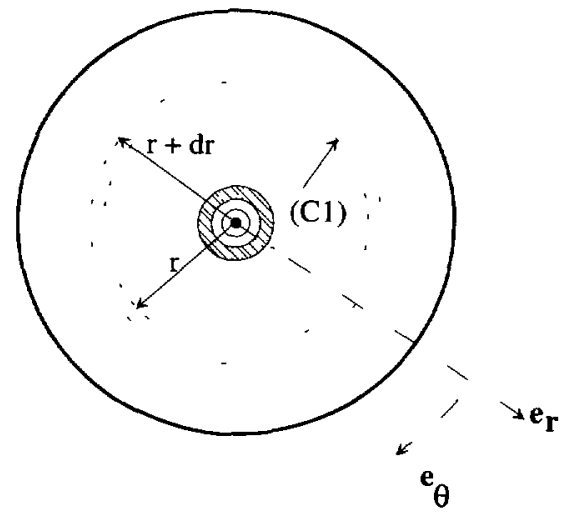

b)

Fig. 1. - Structure du condensateur - a) Coupe. b) Vue de dessus. [Capacitor structure - a) Section. b) Top view.] 
A cause de la symétrie de révolution du condensateur, l'orientation du courant dans les armatures métallisées entre les deux shoopages est donnée par $e_{z}$. Ce courant et celui qui circule dans les conducteurs de connexion créent un champ magnétique. Ce dernier est responsable de l'inductance série équivalente.

L'épaisseur des films couramment utilisés varie de 5 à $12 \mu \mathrm{m}$ pour une tension de service en courant alternatif du condensateur de 200 à $750 \mathrm{~V}$. La hauteur $h$ et le rayon externe $R_{\text {ext }}$ du bobinage peuvent varier de quelques centimètres à une dizaine de centimètres. Le rayon interne $R_{\text {int }}$, pour les condensateurs traditionnels, reste inférieur à $1 \mathrm{~cm}$. La valeur de la capacité dépend de ces divers paramètres et varie de quelques microfarads à quelques dizaines de microfarads.

Les essais présentés dans cet article ont été effectués sur des condensateurs de $9,7 \mu \mathrm{F}$ réalisés avec du film de polypropylène de $10 \mu \mathrm{m}$. La hauteur de ces condensateurs est de $24 \mathrm{~mm}$, leur rayon interne $6 \mathrm{~mm}$ et leur rayon externe $31 \mathrm{~mm}$.

\section{Influence des connexions}

Pour analyser l'importance relative des inductances dues au bobinage et aux connexions, nous utilisons le montage de la figure 2. L'injection d'un courant dans le condensateur est effectuée par les connexions A. La tension est mesurée aux bornes des connexions B (montage de mesure d'impédance 4 points). Le retour du courant se fait, pour ce montage, soit à l'intérieur (Fig. 2a), soit à l'extérieur du condensateur par un conducteur tubulaire qui épouse la forme du condensateur (retour externe, Fig. 2b). Les connexions A et B sont tenues éloignées les unes des autres pour éviter un couplage magnétique entre les circuits.

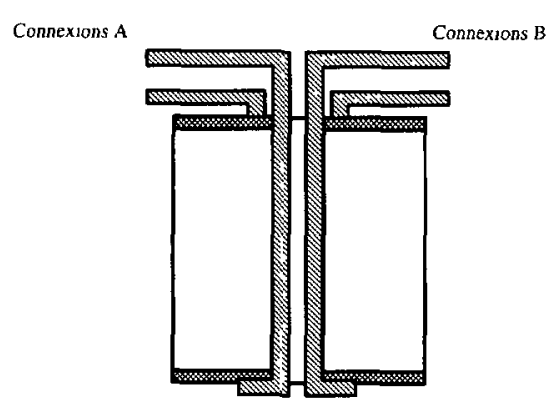

a)

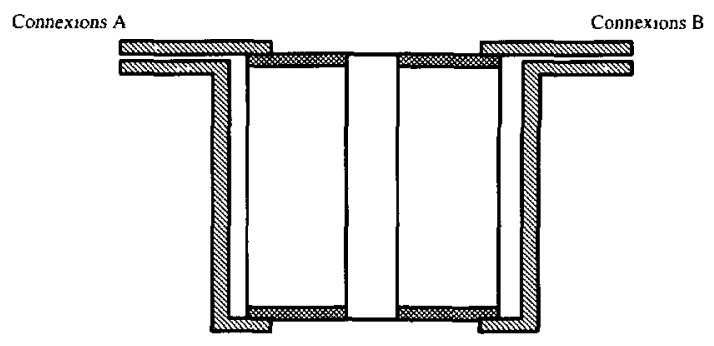

b)

Fig. 2. - Connexion du condensateur - a) Retour interne du courant. b) Retour externe. [Capacitor connection - a) Internal return of current. b) External return.]

L'impédance mesurée par la méthode 4 points décrite précédemment est représentée par des losanges sur les figures $3 a$ (interne) et $3 b$ (externe). Par ailleurs et à titre de comparaison, les cercles (Fig. 3b) représentent l'évolution de l'impédance mesurée de façon traditionnelle avec un montage 2 points (l'injection de courant et la mesure de la tension se font par les mêmes connexions).

Les résultats des méthodes 2 points et 4 points montrent que l'inductance rajoutée par les connexions est importante. Ainsi, pour un montage retour externe, la fréquence de résonance série est de $1,25 \mathrm{MHz}$ pour le bobinage seul et de $275 \mathrm{kHz}$ pour le bobinage plus les connexions. En calculant à partir de ces fréquences de résonance série les valeurs de l'inductance série 


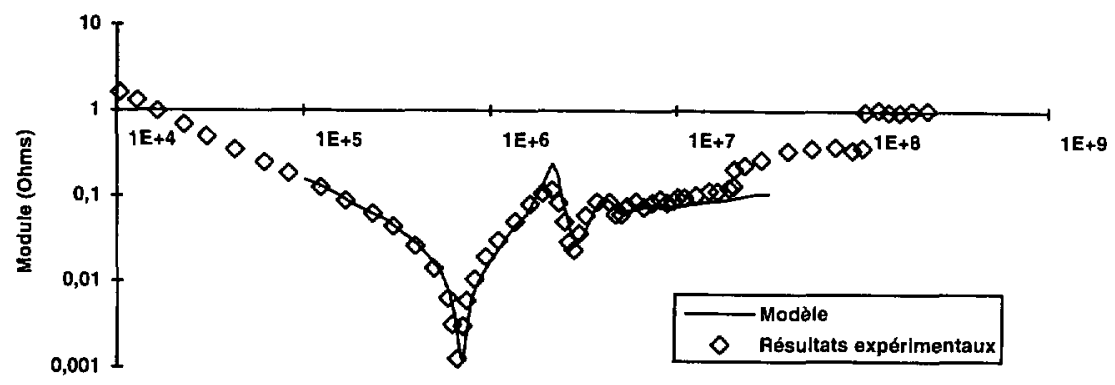

Fréquence (Hz)

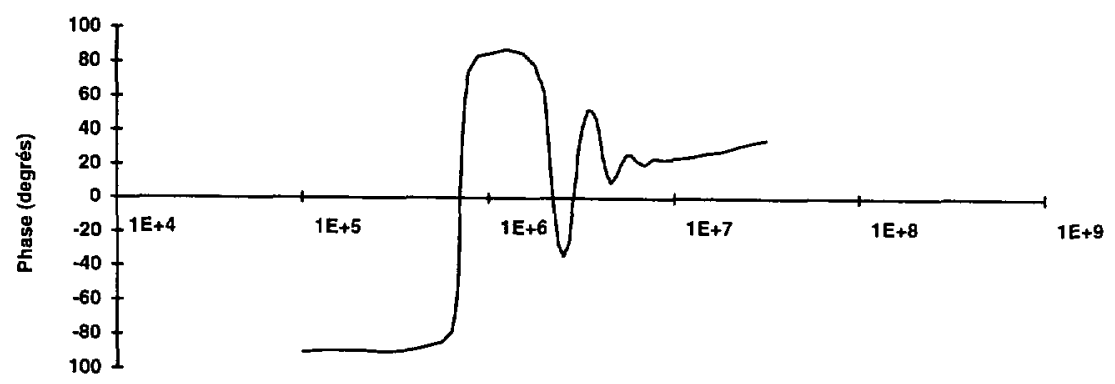

a)

Fréquence (Hz)

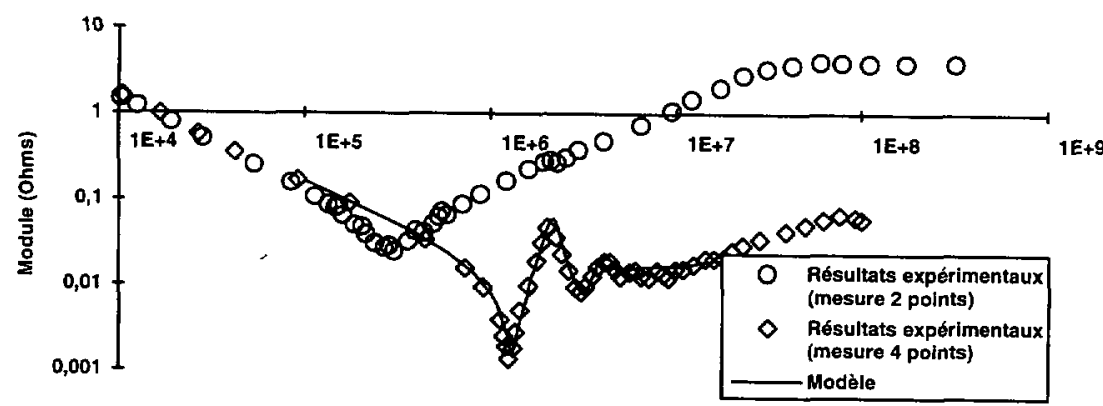

Fréquence (Hz)

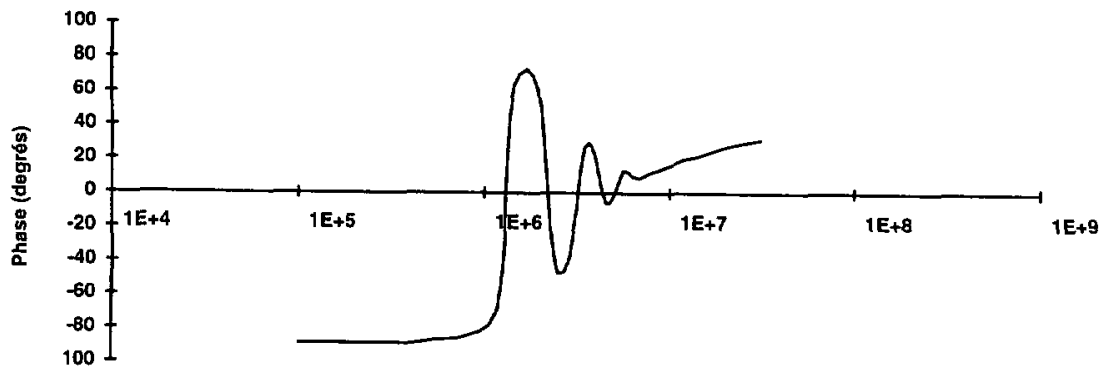

b)

Fréquence $(\mathrm{Hz})$

Fig. 3. - Evolution de l'impédance du condensateur en fonction de la fréquence - a) Retour interne du courant. b) Retour externe. Les valeurs des paramètres pour ce condensateur sont : Capacité, $C=9,7 \mu \mathrm{F}$; hauteur du bobinage, $h=2,4 \mathrm{~cm}$; rayon interne du bobinage, $R_{\text {int }}=0,6 \mathrm{~cm}$; rayon externe du bobinage, $R_{\text {ext }}=3,1 \mathrm{~cm}$; résistance de la métallisation du film, $R_{\square}=8 \Omega$; conductance due aux pertes, $G_{\mathrm{c}}=3,1 \times 10^{5} \Omega^{-1} \mathrm{~m}^{-2}$.

[Evolution of capacitor impedance vs. frequency - a) Internal return of current. b) external return. The values of parameters for this capacitor are: Capacitance, $C=9.7 \mu \mathrm{F}$; height of winding, $h=2.4$ $\mathrm{cm}$; internal radius of winding, $R_{\mathrm{int}}=0.6 \mathrm{~cm}$; external radius of winding, $R_{\text {ext }}=3.1 \mathrm{~cm}$; resistance of 
équivalente du condensateur, nous trouvons une inductance de $1,7 \mathrm{nH}$ pour le bobinage seul et de $35 \mathrm{nH}$ pour le bobinage plus les connexions ; cette dernière valeur est proche de celles mesurées dans les convertisseurs statiques [3]. Par ailleurs, à haute fréquence, l'impédance mesurée avec le montage 2 points est nettement plus grande que celle obtenue avec le montage 4 points ( $40 \mathrm{~dB} \mu \mathrm{V}$ à $20 \mathrm{MHz}$ ). Il apparaît en définitive que l'inductance des connexions peut être bien supérieure à l'inductance du bobinage. Il convient donc, pour limiter les inductances du système, de porter le plus grand soin aux connexions entre les bornes du condensateur et le circuit électrique extérieur ainsi que, à l'intérieur du boîtier, entre le bobinage et les bornes. On peut envisager, par exemple, de réaliser les connexions internes et externes au condensateur en fils plats qui présentent une très faible inductance linéique.

On peut remarquer que l'évolution de l'impédance du bobinage seul fait apparaître des pics à $2 \mathrm{MHz}$ et $4 \mathrm{MHz}$ (pour les plus importants) au-delà de la fréquence de résonance série. Ces pics correspondent à des phénomènes d'antirésonance et de circulation de courants élevés à l'intérieur du condensateur, phénomènes décrits par un modèle proposé par ailleurs [4].

\section{Modèle de répartition du courant et d'impédance}

Pour déterminer l'impédance du condensateur, il est nécessaire de connaître la répartition du courant dans le bobinage et dans le shoopage.

Les variations du courant $\underline{I}(r, \omega)$ orienté suivant $\mathbf{e}_{r}$ qui traverse la boucle $\left(C_{1}\right)$ (Fig. 1b) dépendent $\mathrm{du}$ type de connexions adopté (retour interne ou externe).

Dans le cas d'un retour interne :

$$
\underline{I}\left(r=R_{\mathrm{int}}, \omega\right)=\underline{I}_{c} \quad \underline{I}\left(r=R_{\mathrm{ext}}, \omega\right)=0
$$

Dans le cas d'un retour externe :

$$
\underline{I}\left(r=R_{\text {int }}, \omega\right)=0 \quad \underline{I}\left(r=R_{\text {ext }}, \omega\right)=-\underline{I}_{c} .
$$

En considérant que le champ magnétique est nul à l'extérieur du bobinage, les conditions aux limites sur le shoopage donnent une relation entre le champ magnétique orthoradial $\underline{B}(r, \omega)$ dans le bobinage et le courant $\underline{I}(r, \omega)$ :

$$
\underline{B}(r, \omega)=-\frac{\mu_{0}}{2 \pi r} \underline{I}(r, \omega) .
$$

Cette relation est valable pour les deux configurations.

Le champ magnétique alternatif $\underline{B}(r, \omega)$ va à son tour créer des forces électromotrices et ainsi perturber la répartition de potentiel dans le condensateur. Soit $\underline{V}(r, \omega)$ la différence de potentiel entre deux points, situés à la même distance $r$ de l'axe $\left(O, \mathbf{e}_{z}\right)$, et placés chacun sur un shoopage différent, et $\underline{Z}_{s}(r, \omega) \mathrm{d} r$ l'impédance du shoopage entre deux couronnes circulaires de rayons $r$ et $r+\mathrm{d} r$ (Fig.1b). En appliquant la loi de Faraday, nous obtenons :

$$
\frac{\partial \underline{V}(r, \omega)}{\partial r}=-2 \underline{Z}_{\mathrm{s}}(r, \omega) \underline{I}(r, \omega)+j \omega h \underline{B}(r, \omega) .
$$

La relation précédente montre que le potentiel entre les deux shoopages dépend en général de la distance à l'axe $\left(O, \mathbf{e}_{z}\right)$.

Cette évolution est d'autant plus marquée que le shoopage présente une grande impédance $\left(\underline{Z}_{s}(r, \omega)\right.$ grand) et que le terme $\omega h|\underline{B}(r, \omega)|$ est important. On peut donc prévoir que plus la pulsation $\omega$ sera élevée, moins la répartition de potentiel sera homogène. 
A partir des équations (1) et (2), nous pouvons écrire :

$$
\frac{\partial \underline{V}(r, \omega)}{\partial r}=\left(\frac{4 \pi r}{\mu_{0}} \underline{Z}_{s}(r, \omega)+j \omega h\right) \underline{B}(r, \omega) .
$$

En basse fréquence, l'impédance du "shoopage" entre deux couronnes circulaires de rayons $r$ et $r+\mathrm{d} r$ est résistive et a pour expression :

$$
\underline{Z}_{\mathrm{s}}(r, \omega) \mathrm{d} r=\rho_{\mathrm{s}} \frac{\mathrm{d} r}{2 \pi r e_{\mathrm{s}}}=\frac{\underline{z}_{\mathrm{s}}}{2 \pi r} \mathrm{~d} r
$$

avec $\underline{z}_{\mathrm{s}}=\frac{\rho_{\mathrm{s}}}{e_{\mathrm{s}}}$, où $\rho_{\mathrm{s}}$ représente la résistivité du shoopage, $\mathrm{d} r$ la longueur des lignes de courant, $e_{\mathrm{s}}$ l'épaisseur du "shoopage" et $2 \pi r e_{\mathrm{s}}$ la surface de la section traversée par le courant.

Pour une fréquence élevée, quand l'épaissseur de peau $\delta_{\mathrm{s}}$ dans le shoopage devient faible par rapport à l'épaisseur du shoopage, l'impédance devient :

$$
\underline{Z}_{\mathrm{s}}(r, \omega) \mathrm{d} r=\frac{(1+j) \rho_{\mathrm{s}}}{\delta_{\mathrm{s}}} \times \frac{\mathrm{d} r}{2 \pi r}=\frac{\underline{z}_{\mathrm{s}}}{2 \pi r} \mathrm{~d} r
$$

avec $\underline{z}_{\mathrm{s}}=\frac{(1+j) \rho_{\mathrm{s}}}{\delta_{\mathrm{s}}}$

Le paramètre $\underline{Z}_{\mathrm{s}}(r, \omega)$ peut donc s'écrire sous la forme :

$$
\underline{Z}_{\mathrm{s}}(r, \omega)=\frac{\underline{z}_{\mathrm{s}}(\omega)}{2 \pi r}
$$

où $\underline{z}_{\mathrm{s}}(\omega)$ dépend de la nature et de l'épaisseur du "shoopage" ainsi que de la pulsation des courants mais ne dépend pas du paramètre géométrique $r$.

Les simulations ont été effectuées en prenant comme expression de $\underline{z}_{s}$ :

$$
\underline{z}_{\mathrm{s}}=\frac{(1+j) \rho_{\mathrm{s}}}{\delta_{\mathrm{s}}}
$$

avec $\delta_{\mathrm{s}}=\sqrt{\frac{2 \rho_{\mathrm{s}}}{\mu_{\mathrm{s}} \omega}}$, où $\mu_{\mathrm{s}}$ représente la perméabilité magnétique du "shoopage". Par ailleurs, on a considéré que le shoopage était en zinc, ce qui revient à prendre comme valeurs de $\mu_{\mathrm{s}}$ et de $\rho_{\mathrm{s}} \cdot \mu_{\mathrm{s}}=4 \pi \times 10^{-7} \mathrm{Hm}^{-1} \rho_{\mathrm{s}}=5,8 \times 10^{-8} \Omega \mathrm{m}$.

A partir des équations (3) et (6), on trouve :

$$
\frac{\partial \underline{V}(r, \omega)}{\partial r}=\left(\frac{2 \underline{z}_{s}(\omega)}{\mu_{0}}+j \omega h\right) \underline{B}(r, \omega) .
$$

Nous pouvons par ailleurs lier la répartition de potentiel entre les deux "shoopages" à la densité surfacique de courant $\underline{J}_{s}(r, \omega)$ qui traverse le condensateur suivant $\mathbf{e}_{z}$. Si on note $\underline{G}_{s}(\omega) \mathrm{d} S$ la conductance entre deux portions de shoopage en regard, on peut écrire :

$$
\underline{J}_{s}(r, \omega)=\underline{G}_{s}(\omega) \underline{V}(r, \omega) .
$$

Soit :

$$
\gamma=\frac{C}{\pi\left(R_{\mathrm{ext}}^{2}-R_{\mathrm{int}}^{2}\right)}
$$

la capacité par unité de surface du "shoopage" ; la conductance par unité de surface $\underline{G}_{s}(\omega)$ correspond à la mise en série d'une conductance $j \gamma \omega$ due à l'effet capacitif et d'une conductance 
$G_{\mathrm{c}}$ due aux pertes diélectriques et aux pertes par effet Joule dans la métallisation [5]. On obtient comme expression de $\underline{G}_{\mathbf{s}}(\omega)$ :

$$
\underline{G}_{s}(\omega)=\frac{j \gamma \omega}{1+j \frac{\gamma \omega}{G_{c}}}
$$

Pour le condensateur étudié, $G_{\mathrm{c}}=3,1 \times 10^{5} \Omega^{-1} \mathrm{~m}^{-2}$.

La densité surfacique de courant $\underline{J}_{s}(r, \omega)$ est par ailleurs liée au courant $\underline{I}$ par la relation :

$$
\frac{\partial \underline{I}(r, \omega)}{\partial r}=-2 \pi r \underline{J}_{s}(r, \omega)
$$

où le terme $2 \pi r \underline{J}_{s}(r, \omega)$ représente le courant qui traverse le bobinage à une distance du centre comprise entre $r$ et $r+\mathrm{d} r$.

En combinant les équations (8), (9) et (12), nous pouvons écrire l'équation de répartition du champ magnétique dans le condensateur.

Nous posons pour simplifier :

$$
\delta_{\mathrm{c}}^{2}=\frac{-1}{2 \underline{G}_{\mathrm{s}}(\omega) \underline{z}_{\mathrm{s}}+j \omega h \mu_{0} \underline{G}_{\mathrm{s}}(\omega)}
$$

ou encore :

$$
\delta_{c}=\left[\frac{-1}{\underline{G}_{s}(\omega)\left[2 \underline{z}_{s}+j \omega h \mu_{0}\right]}\right]^{1 / 2}
$$

Nous obtenons alors l'équation :

$$
r^{2} \frac{\partial^{2} \underline{B}(r, \omega)}{\partial r^{2}}+r \frac{\partial \underline{B}(r, \omega)}{\partial r}+\left[\frac{r^{2}}{\delta_{\mathrm{c}}^{2}}-1\right] \underline{B}(r, \omega)=0
$$

L'équation (14) a la même forme que celle qui régit la répartition du champ magnétique dans un conducteur cylindrique. Le coefficient $\delta_{\mathrm{c}}$ est ainsi analogue à l'épaisseur de peau dans les conducteurs, mais son expression est, bien sûr, différente.

Pour résoudre l'équation (14), nous notons $J_{n}$ et $Y_{n}$ les fonctions de Bessel d'ordre $n$ respectivement de première et de deuxième espèce.

En tenant compte des conditions aux limites, les solutions de l'équation (14) s'écrivent [6] : - pour un retour au centre :

$$
\underline{B}_{\mathrm{i}}(r, \omega)=\frac{\mu_{0} \underline{I}_{\mathrm{c}}}{2 \pi R_{\mathrm{int}} \Delta}\left[Y_{1}\left(\frac{R_{\mathrm{ext}}}{\delta_{\mathrm{c}}}\right) J_{1}\left(\frac{r}{\delta_{\mathrm{c}}}\right)-J_{1}\left(\frac{R_{\mathrm{ext}}}{\delta_{\mathrm{c}}}\right) Y_{1}\left(\frac{r}{\delta_{\mathrm{c}}}\right)\right]
$$

- pour un retour externe :

$$
\underline{B}_{\mathrm{e}}(r, \omega)=\frac{\mu_{0} \underline{I}_{\mathrm{c}}}{2 \pi R_{\mathrm{int}} \Delta}\left[Y_{1}\left(\frac{R_{\text {int }}}{\delta_{\mathrm{c}}}\right) J_{1}\left(\frac{r}{\delta_{\mathrm{c}}}\right)-J_{1}\left(\frac{R_{\text {int }}}{\delta_{\mathrm{c}}}\right) Y_{1}\left(\frac{r}{\delta_{\mathrm{c}}}\right)\right]
$$

où :

$$
\Delta=J_{1}\left(\frac{R_{\text {ext }}}{\delta_{\mathrm{c}}}\right) Y_{1}\left(\frac{R_{\mathrm{int}}}{\delta_{\mathrm{c}}}\right)-J_{1}\left(\frac{R_{\mathrm{int}}}{\delta_{\mathrm{c}}}\right) Y_{1}\left(\frac{R_{\mathrm{ext}}}{\delta_{\mathrm{c}}}\right)
$$

D'autre part, les équations (1) et (12) permettent de déterminer la densité de courant dans le condensateur :

- pour un retour au centre :

$$
\underline{J}_{\mathrm{si}}(r, \omega)=\frac{\underline{I}_{\mathrm{c}}}{2 \pi R_{\mathrm{int}} \delta_{\mathrm{c}} \Delta}\left[Y_{1}\left(\frac{R_{\mathrm{ext}}}{\delta_{\mathrm{c}}}\right) J_{0}\left(\frac{r}{\delta_{\mathrm{c}}}\right)-J_{1}\left(\frac{R_{\mathrm{ext}}}{\delta_{\mathrm{c}}}\right) Y_{0}\left(\frac{r}{\delta_{\mathrm{c}}}\right)\right]
$$


- pour un retour externe :

$$
\underline{I}_{\mathrm{se}}(r, \omega)=\frac{\underline{I}_{\mathrm{c}}}{2 \pi R_{\mathrm{int}} \delta_{\mathrm{c}} \Delta}\left[Y_{1}\left(\frac{R_{\text {int }}}{\delta_{\mathrm{c}}}\right) J_{0}\left(\frac{r}{\delta_{\mathrm{c}}}\right)-J_{1}\left(\frac{R_{\mathrm{int}}}{\delta_{\mathrm{c}}}\right) Y_{0}\left(\frac{r}{\delta_{\mathrm{c}}}\right)\right]
$$

On peut remarquer que cette densité de courant dépend en partie du rapport entre le rayon extérieur du condensateur et la "pseudo épaisseur de peau" $\delta_{\mathrm{c}}$.

L'impédance totale du condensateur $\left(\underline{Z}_{\text {tot }}\right)$ se déduit directement de l'expression de la répartition de la densité de courant dans le condensateur (17), $l_{\mathrm{s}}$ et $r_{\mathrm{s}}$ représentant respectivement l'inductance et la résistance des connexions du condensateur. $\underline{Z}_{\text {tot }}$ se met sous la forme :

$$
\underline{Z}_{\mathrm{tot} \mathrm{i}}=\frac{\underline{J}_{\mathrm{si}}\left(R_{\text {int }}, \omega\right)}{\underline{G}_{\mathrm{s}}(\omega) \underline{I}_{\mathrm{c}}}+j l_{\mathrm{s}} \omega+r_{\mathrm{s}} \quad \text { pour un retour interne ; }
$$

et

$$
\underline{Z}_{\mathrm{tot} \mathrm{e}}=\frac{\underline{J}_{\mathrm{se}}\left(R_{\mathrm{ext}}, \omega\right)}{\underline{G}_{\mathrm{s}}(\omega) \underline{I}_{\mathrm{c}}}+j l_{\mathrm{s}} \omega+r_{\mathrm{s}} \quad \text { pour un retour externe. }
$$

Les termes $l_{\mathrm{s}}$ et $r_{\mathrm{s}}$ peuvent être négligés dans le cas d'une mesure d'impédance 4 points.

Pour les faibles fréquences, le paramètre $\delta_{\mathrm{c}}$ devient grand par rapport aux dimensions du condensateurs $R_{\text {int }}$ et $R_{\text {ext }}$. Les variables des fonctions de Bessel dans les équations (17a et b) tendent alors vers 0 .

En effectuant un développement de ces fonctions au voisinage de zéro et en reportant les expressions de $\underline{J}_{s}(r, \omega)$ ainsi obtenues dans les équations (18a et b) on trouve :

$$
\begin{aligned}
\underline{Z}_{\text {tot } \mathrm{i}}=\underline{Z}_{\mathrm{tot} \mathrm{e}}=\underline{Z}_{\mathrm{tot}} & =\frac{1}{\pi\left(R_{\mathrm{ext}}^{2}-R_{\mathrm{int}}^{2}\right) \times j \gamma \omega}+j l_{\mathrm{s}} \omega+r_{\mathrm{s}} \\
& =\frac{1}{j C \omega}+j l_{\mathrm{s}} \omega+r_{\mathrm{s}}
\end{aligned}
$$

On retrouve ainsi l'impédance du condensateur à basse fréquence.

Les pics observés sur les figures $3 a$ et $3 b$ correspondent aux maxima de densité de courant et donc aux minima de l'expression $\Delta$.

La variation de l'impédance donnée par le modèle est représentée en traits pleins sur les figures $3 \mathrm{a}$ et $3 \mathrm{~b}$. La fréquence $f_{\mathrm{a}}$ des phénomènes d'antirésonance observés est pratiquement indépendante du mode de connexion (retour interne ou externe). En revanche, la fréquence de résonance série $f_{\mathrm{r}}$ est plus faible quand le retour du courant a lieu à l'intérieur (Fig. 3a). Ce fait est dû à la répartition du champ magnétique $\mathbf{B}$ dans le condensateur. En effet, dans le cas d'un retour interne, le champ magnétique à l'intérieur du condensateur (Fig. 4) est plus important que celui observé dans le cas d'un retour externe. En conséquence, l'énergie magnétique emmagasinée dans le condensateur est plus forte et la fréquence de résonance diminue. De façon générale, pour diminuer le champ magnétique, à courant constant et à perméabilité constante, on peut augmenter la longueur des lignes de champ, c'est-à-dire faire circuler les courants inducteurs à la périphérie du condensateur. L'orientation de notre travail va donc porter sur des géométries qui permettent d'éloigner les courants du centre du condensateur.

\section{Influence de la géométrie}

Afin de déterminer l'influence des paramètres géométriques, des simulations de divers types de condensateurs ont été effectuées. Pour que les résultats soient comparables, le volume du bobinage et la capacité ont été maintenus constants (égaux respectivement à $6,97 \times 10^{-5} \mathrm{~m}^{3}$ 


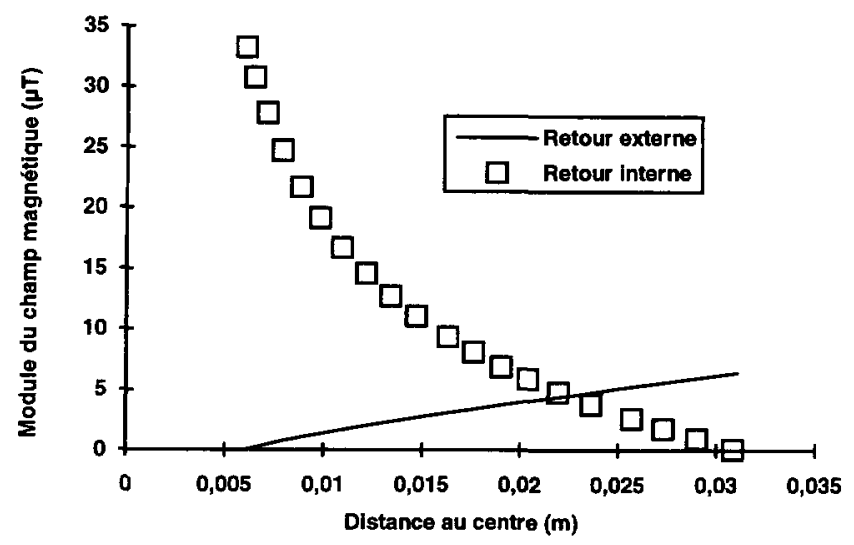

Fig. 4. - Répartition du champ magnétique dans le condensateur à $200 \mathrm{kHz}$. [Distribution of magnetic field in capacitor at $200 \mathrm{kHz}$.]

et $9,7 \mu \mathrm{F})$. Pour chaque géométrie de bobinage, - caractérisée par sa hauteur $h$ et ses rayons interne et externe $R_{\text {int }}$ et $R_{\text {ext }}$ respectivement - nous relevons la fréquence de résonance série $f_{\mathrm{r}}$ correspondant au minimum d'impédance ainsi que la fréquence $f_{\mathrm{a}}$ correspondant au premier minimum relatif de phase après $f_{\mathbf{r}}$ (Fig. 3 ).

Ces condensateurs peuvent être rangés dans deux "familles" : les condensateurs plats (à faible hauteur $h$ ) et les condensateurs annulaires (à rayon interne du bobinage $R_{\text {int }}$ important).

4.1. Condensateur plat. - L'évolution des fréquences de résonance et d'antirésonance du condensateur est représentée, sur la figure 5, pour les deux modes de connexion (interne et externe) en fonction du rayon externe du condensateur. On remarque que les fréquences de résonance et d'antirésonance augmentent avec le rayon extérieur. Il apparaît que, quelle que soit la géométrie du condensateur :

- la fréquence d'antirésonance dépend peu du mode de connexion ;

- la fréquence de résonance avec retour interne du courant est inférieure à la fréquence de résonance avec retour externe et augmente moins vite quand le rayon externe croît.

On peut expliquer que la fréquence d'antirésonance dépend peu du mode de connexion en remarquant que le volume du condensateur compris entre $R_{\text {int }}$ et $R_{\text {ext }}$ se comporte comme une cavité cylindrique résonante d'hyperfréquence [7], l'amortissement étant cependant supérieur. En effet, pour un mode donné, la fréquence de cette dernière est fixée par la seule géométrie de la cavité. Par ailleurs, la présence sur la figure 3 b d'un second pic d'antirésonance à 4,1 $\mathrm{MHz}$ correspond, pour une cavité hyperfréquence, à un second mode de résonance. Cependant, les pics d'antirésonance de fréquence supérieure, qui correspondent aussi aux minima de $\Delta$, n'apparaissent plus à cause des pertes dans le bobinage, très supérieures aux pertes dans une cavité hyperfréquence.

4.2. Condensateur annulaire. - La figure 6 représente l'évolution des fréquences de résonance et d'antirésonance pour un condensateur annulaire dans les deux modes de connexion. Comme pour le condensateur plat, la fréquence d'antirésonance est quasi indépendante du mode de connexion et la fréquence de résonance est plus grande quand le retour du courant se fait à l'extérieur. Cependant, à la différence du condensateur plat, l'écart des fréquences de 


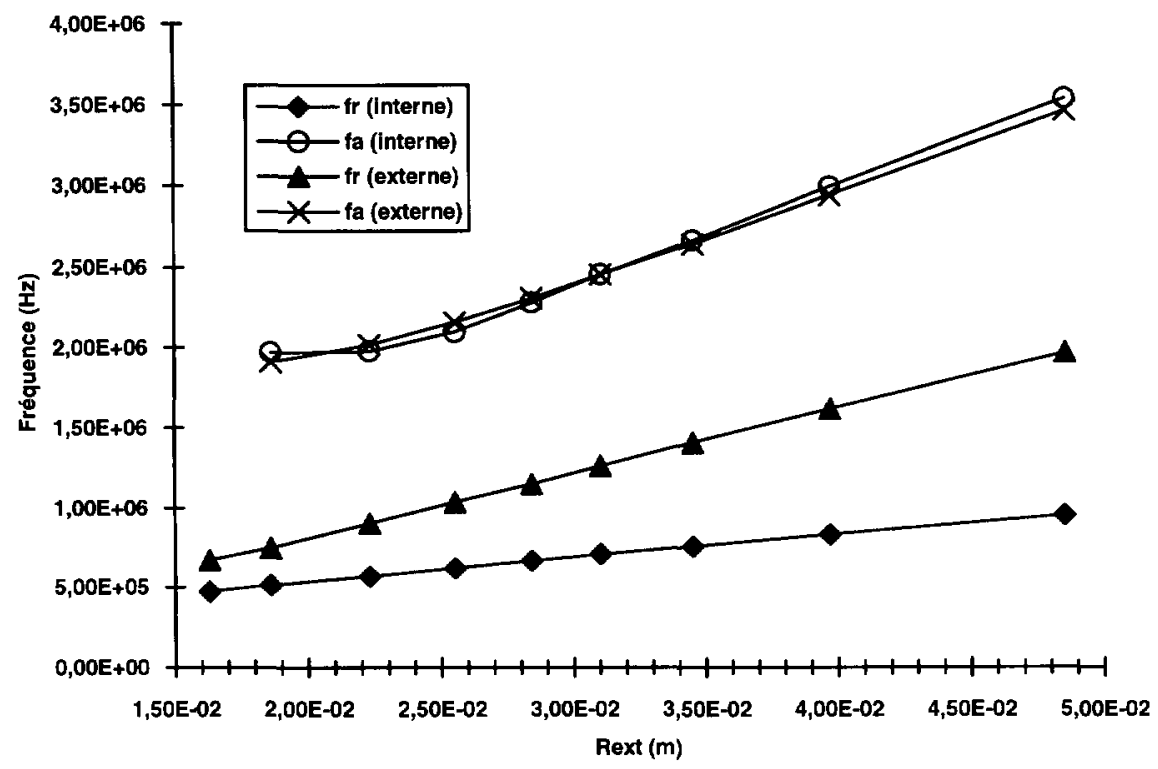

Fig. 5. - Fréquences de résonance et d'antirésonance dans un condensateur plat. [Resonant and antiresonant frequencies in a flat capacitor.]

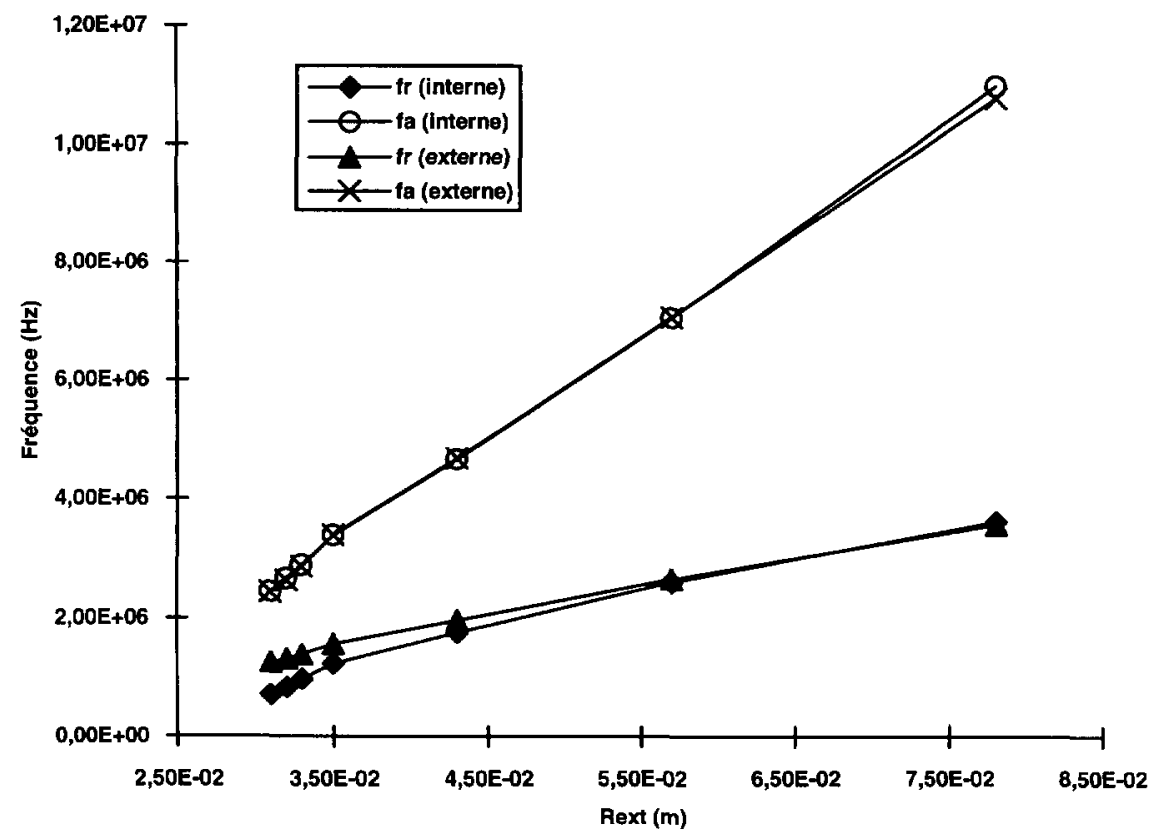

Fig. 6. - Fréquences de résonance et d'antirésonance dans un condensateur annulaire. [Resonant and antiresonant frequencies in an annular capacitor.] 


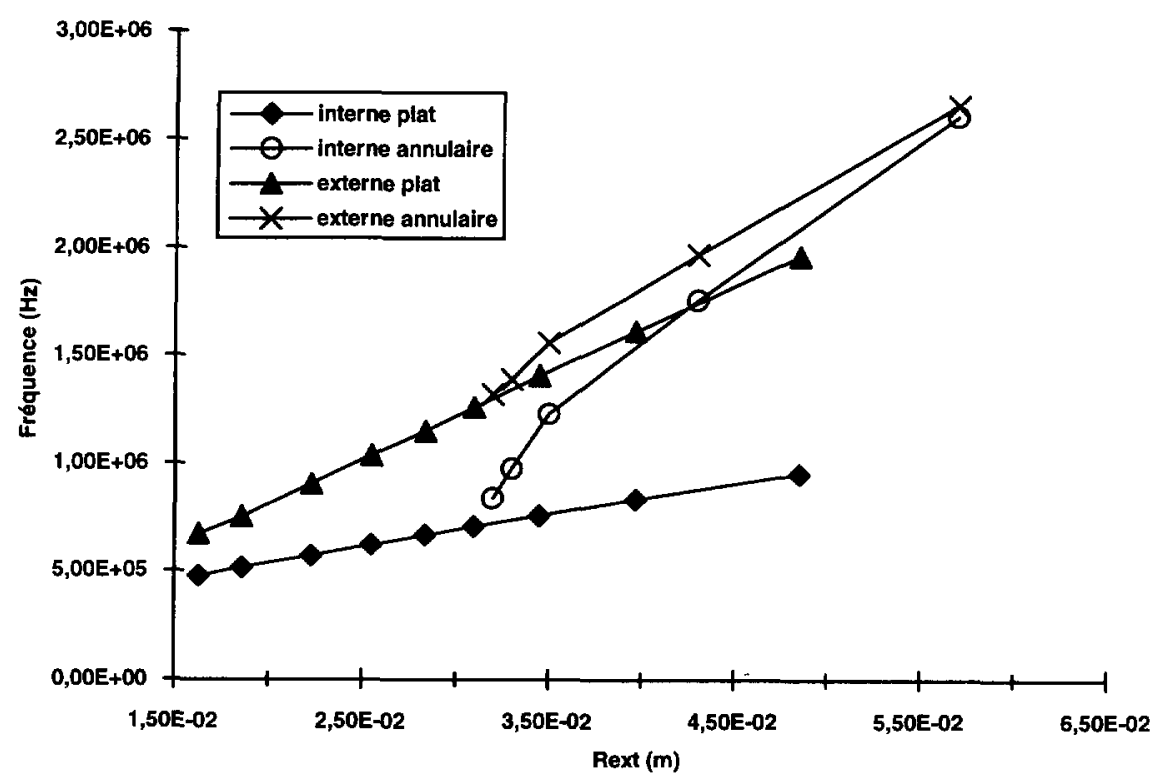

Fig. 7. - Fréquences de résonance dans un condensateur pour diverses géométries en fonction du rayon externe.

[Resonant frequencies in a capacitor for various shapes vs. the external radius.]

résonance pour les deux modes de connexion diminue quand le rayon externe du condensateur augmente. Ceci est dû au fait que la différence entre rayons interne et externe du condensateur diminue pour garder un volume de bobinage constant et que, par conséquent, la différence entre un retour interne et un retour externe du courant s'estompe.

D'autre part, comme on peut le constater sur la figure 7 , la fréquence de résonance série est plus grande, à même rayon externe, pour un condensateur annulaire que pour un condensateur plat. Pour une même géométrie, elle est aussi plus grande avec un retour externe du courant. Il convient donc, pour diminuer l'inductance propre du condensateur, d'employer des condensateurs de géométrie annulaire, de grands rayons externe et interne et d'effectuer la connexion de retour du courant à l'extérieur du condensateur.

\section{Conclusion}

Les mesures d'impédance effectuées sur les condensateurs et l'exploitation d'un modèle théorique nous ont permis de mettre en avant l'importance de l'inductance des connexions. Celle-ci peut en effet être plusieurs dizaines de fois supérieure à celle du bobinage seul pour un montage peu soigné. Il ressort, des simulations d'impédance présentées par des bobinages de géométries diverses, que les bobinages plats ou, mieux encore, annulaires, à grands rayons externe et interne, présentent une fréquence de résonance élevée. De façon générale, les connexions de retour du courant doivent passer à l'extérieur du bobinage pour réduire l'inductance série équivalente du condensateur. 


\section{Bibliographie}

[1] Sarjeant W.J., Capacitors, IEEE Trans. Elec. Insul. 25 (1990) 861-922.

[2] Mouriès G., Condensateurs utilisés en électronique de puissance, Tech. Ing. (1980) D-3-I, D3280.

[3] Costa F., Forest F., Puzo A. et Rojat G., Etude des perturbations conduites et rayonnées dans une cellule de commutation, J. Phys. III France 3 (1993) 2221-2248.

[4] Joubert Ch., Béroual A. et Rojat G., Magnetic Field and Current Distribution in Metallized Capacitors, J. Appl. Phys. 76 (1994).

[5] Joubert $\mathrm{Ch}$., Rojat G. et Béroual A., "Influence de la fréquence sur les pertes dans les condensateurs métallisés", Cinquième Colloque National, Electronique de Puissance du Futur (Cachan 28 - 30 septembre 1994).

[6] Abramowitz M. et Stegun I.A., Handbook of Mathematical Functions (Dover Publications Inc., New York, 1972).

[7] Feynman R.P., Leighton R.B. et Sands M., The Feynman Lectures on Physics, Tome II, Chap. 23 (Addison-Wesley Publishing Compagny, 1967). 